\title{
MOLECULAR DOCKING STUDIES OF SNAKE VENOM SERINE PROTEASE OF SHARP-NOSED PIT VIPER WITH HESPERETIN
}

\author{
SUBHAMAY PANDA ${ }^{1,2 *}$, IMAN EHSAN ${ }^{3}$ \\ ${ }^{1}$ Department of Pharmacy, Gupta College of Technological Sciences, Ashram More, G.T. Road, Asansol - 713 301, West Bengal, India. ${ }^{2}$ Department \\ of Biological Sciences, Indian Institute of Human and Social Sciences, Sitarampur, Asansol - 713 359, West Bengal, India. ${ }^{3}$ Department of \\ Pharmaceutical Technology, Jadavpur University, Kolkata - 700 032, West Bengal, India. Email: Subhamay_panda@rediffmail.com
}

Received: 28 February 2018, Revised and Accepted: 17 April 2018

ABSTRACT

Objective: The management of snake bite envenomation is a global challenge affecting millions of people. Immunotherapy is still regarded as the treatment of choice; however, their subsequent adverse effects restrict their potential use for therapy against snake venom poisoning. In recent years, more attention has been given to the exploration of indigenous medicinal plants for their outstanding benefits for the treatment of several diseases and disorders, including snake venom poisoning. Hesperetin is a naturally occurring compound derived from a flavanone glycoside, hesperidin and is obtained from various citrus fruits. It is known to possess significant inhibitory activity against snake venom serine proteases. The aim of our present study was to investigate the significant inhibitory action of hesperetin on thrombin-like serine protease from sharp-nosed pit viper (Deinagkistrodon acutus) snake venom.

Methods: We have employed molecular docking analysis by implementing the state-of-the-art docking program to validate the hypothesis of the prospective inhibitory properties of hesperetin on thrombin-like serine proteases of snake venom. AutoDock 4.2, InterProSurf, MGLTools, and MTiAutoDock were utilized for the molecular docking analysis of thrombin-like serine protease obtained from the snake venom of sharp-nosed pit viper with the natural compound hesperetin.

Results: The results generated from in silico based approach reveals the significant inhibitory role of hesperetin against thrombin-like snake venom proteases, which might lead to the drug designing of the inhibitors of snake venom serine proteases.

Conclusion: The implementation of molecular docking analysis by the employment of state-of-the-art docking program supports the potential of inhibitory activity of naturally obtained hesperetin compound on thrombin-like serine proteases of snake venom. The generated in silico results suggests that the novel structure hesperetin - flavanone might act as a potent inhibitor of thrombin-like snake venom proteases, and unlocks the possibilities for designing drugs of the inhibitors of snake venom serine proteases. Moreover, the investigation of the novel compound obtained from natural sources for their inhibitory activity against snake venom serine proteases would lead to the discovery of newer inhibitory compound from a highly uninvestigated research arena.

Keywords: Snake venom, Serine protease, Sharp-nosed pit viper, Deinagkistrodon acutus, Hesperetin, Molecular docking

(C) 2018 The Authors. Published by Innovare Academic Sciences Pvt Ltd. This is an open access article under the CC BY license (http://creativecommons. org/licenses/by/4. 0/) DOI: http://dx.doi.org/10.22159/ajpcr.2018.v11i6.25531

\section{INTRODUCTION}

The poisonous snakes utilize their venom to immobilize or kill their prey and digest them and also for the purpose of defense. During the case of snakebite, the venom gets injected into the body of a victim through their pointed fangs, and consequently, it might lead to injury in numerous ways. The snake venoms mainly cause an effect on the crucial elements of the physiological pathway of every animal. For instance, neurotoxins cause blockade in the nerve-muscle junction; phospholipases and cytotoxins cause necrosis of muscle fibers; cobra venom factor inhibits numerous proteins and circulating complements including the activity of enzymes which lead to systemic hemostatic defects. The components of venom appear to be quite similar and interrelated among each family of snakes [1,2]. The venom glands of snakes are enriched with a variety of proteolytic enzymes such as serine proteinases and metalloproteinases which play a very crucial activity in the envenomation of mammals. The serine proteinases of snake venom make up a group of widely studied toxins which mostly affects the hemostatic system. They are generally located in the terrestrial snake venoms belonging to the families Crotalidae, Viperidae, Colubridae, and Elapidae, and very often in the sea snakes (Hydrophiidae) venom $[3,4]$.

Most of the venoms contain proteases and are investigated by several researchers since the emergence of this century when the first evidence of snake venom-induced blood coagulation was observed. The purification of first snake venom protease possessing blood clotting potential was done in the 1950s. Till date, the isolated proteases are categorized on the basis of their structures into following two groups: (i) Serine proteases and (ii) metalloproteinases. Only a few evidences are found for the existence of aspartic proteases and thiol proteases in snake venoms. Among these proteases, few of them are known for degradation of tissue proteins of mammals in the bite area nonspecifically to paralyze the victims. However, many of these proteases lead to the cleavage of victims' plasma proteins specifically to yield either the potent activators or inhibitors that affect their physiological processes such as hemostasis and thrombosis, leading to blood clotting, platelet aggregation, and fibrinolysis [5-7].

Hesperidin is one of the major flavonoid glycoside naturally present in several citrus fruits such as lemons and oranges [8]. The aglycone form of hesperidin is known as hesperetin, which is originated from the word "hesperidium," the type of fruit grown in citrus plants. In the year 1828, a French chemist Lebreton was the first to isolate hesperidin from the white inner layer of peels (albedo and mesocarp) of citrus fruits. Hesperidin is known to play a significant role in defense of plants $[8,9]$. The modern state-of-the-art bioinformatical research approaches make it's quite suitable to solve several complicated issues related to medical 
science, pharmaceutical science, and biological science [10-17]. In recent years, literatures have reported that hesperetin possesses the potential to behave as an effective inhibitor of thrombin-like serine proteases isolated from the venom of Crotalus simus snakes [18]. The objective of the present research work was to explore the inhibitory activity of hesperetin on thrombin-like serine protease obtained from sharp-nosed pit viper (Deinagkistrodon acutus) venom. We have implemented molecular docking analysis by employing the state-ofthe-art docking program to establish the hypothesis of the potential inhibitory activity of hesperetin on thrombin-like serine proteases of snake venom. Therefore, our effort was to investigate the novel compound procured from natural sources for its inhibitory activity against snake venom serine proteases to identify newer inhibitory compound from a highly uninvestigated research area.

\section{MATERIALS AND METHODS}

The amino acid sequence of thrombin-like serine protease from sharpnosed pit viper snake venom was collected from the online database system of National Centre for Biotechnology Information (http:// ncbi.nlm.nih.gov) [19]. Signal P 4.0 online server was employed for detecting an instance of the signal peptide in the protein sequence of thrombin-like serine protease [20]. The generation of the structure of thrombin-like serine protease from the venom of sharp-nosed pit viper was carried out by the technique described in research work by Panda et al. $[11,17]$. Briefly, the Iterative Threading ASSEmbly Refinement (I-TASSER) suite is a strategy of hierarchical modeling to the structure of proteins and also to predict their active function on account of the secondary structure of protein amplified profile-profile threading alignment and the conceptual implementation of the TASSER program [21]. Eventually, the structure of I-TASSER created molecular model was further improved by energy minimization measure through GROMOS 96 43B1 parameters set [22]. Later, the model of threedimensional structure was established and evaluated by PROCHECK tool and ProSA-web tool $[23,24]$. UCSF Chimera package was implemented for analyzing the hydrophobicity molecular surface [25]. AutoDock 4.2, InterProSurf, MGLTools, and MTiAutoDock were utilized for the molecular docking analysis of thrombin-like serine protease obtained from the snake venom of sharp-nosed pit viper with the natural compound hesperetin [26-30].

\section{RESULTS AND DISCUSSION}

The envenomation by snake bites necessitates urgent medical attention. Therefore, the beneficial novel lead compounds whose identification might lead to the development of an effective and functional affordable complementary treatment for improving the treatment strategies of snakebite poisoning might lead to the development of inhibitors more impressive [31]. The compounds obtained from natural sources for the inhibition of snake venom toxins plays a very powerful role in neutralizing the detrimental activities of snake venoms. Numerous researches are reported that shows the utilization of traditional medicinal plants for the treatment of snake bite poisoning. A plethora of medicinal plants is reported that possess the potential to combat the effect of snake venom [32]. According to numerous investigations, the presence of several essential phytoconstituents in these medicinal plants is responsible for an effective role in snakebite envenomation. Several endeavors are carried out for investigating these indigenous medicinal plants and for identifying their chief active components and significant therapeutic benefits. The potential phytoconstituents obtained from medicinal plants bears numerous pharmacological properties and might provide an outstanding contribution as effective therapeutic agents for snakebite treatment [33-35]. In this research paper, computational molecular docking investigations were carried out to evaluate the likelihood of inhibition of thrombin-like serine protease being by flavonoid glycosides. To determine initial structural quality, we have implemented Ramachandran plot analysis. For the purpose of validation, Ramachandran plot analysis by utilizing total score of G-factor (PROCHECK analysis) is an excellent standard. Fig. 1.
Illustrates the Ramachandran plot for thrombin-like serine protease obtained from sharp-nosed pit viper snake venom.

Overall, $100 \%$ of the residues were observed in allowed as well as favored regions, which help in validating the standard of generated structural protein model as shown in Fig. 2. The total score of G-factor observed for thrombin-like serine protease of sharp-nosed pit viper was known to be -0.39 that was quite higher than the acceptable value of -0.50 . Hence, it is proposed that the modeled structure of thrombinlike serine protease of sharp-nosed pit viper snake venom is quite acceptable.

ProSA-web was utilized to explore three-dimensional structural models of thrombin-like serine protease of sharp-nosed pit viper for detecting any errors. It was observed that the Z-score of thrombin-like serine protease of sharp-nosed pit viper was -7.64 , as depicted in Fig. 3. The obtained score was within the scores range as seen for identical sized proteins, thereby specifying extremely reliable structures. The hydrophobicity molecular surface analysis (dodger blue shown for the most hydrophilic, to white, to orange-red for the most hydrophobic) that exhibits the surface of the molecules, colored by hydrophobicity of amino acid, portrays that the hydrophobicity surface specific to molecules is distinctive to serine protease molecule of sharp-nosed pit viper venom, as illustrated in Fig. 4.

In the current research work, the docking of inhibitor hesperetin was done with thrombin-like serine protease isolated from the venom of sharp-nosed pit viper by employing the AutoDock program. The cleaning and automatic preprocessing of the structure of thrombinlike serine protease of sharp-nosed pit viper was attained where entire HETATMs are discarded, and the addition of hydrogen atoms to the structure was done by employing MGLTools (17). Fig. 5. displays the docked molecules exhibiting hesperetin binding with thrombin-like serine protease of sharp-nosed pit viper having the binding energy of -5.450000 . Among the 10 binding conformations, the selection of ideal pose was done on account of binding energy calculations (Table 1). Overall, 10 docking runs are carried out, bearing total initial population of 150 random individuals. Apart from this, InterProSurf server was employed to predict the overall attainable surface area (as 9745.91) of the docked structure where polar area/energy was detected as 3119.74 , and a polar area/energy was detected as 6626.18 having 1.4 prob radiuses. The estimation of the total number of surface atoms was considered to be 928 while the total buried atoms were predicted to be 855 . The ideal ligand binding pose of hesperetin which is in close proximity to catalytic residues of snake venom serine protease, i.e., serine, histidine, and aspartic acid reveal that hesperetin could interact with nearby residues and form bonds. The obtained results could recommend that the hesperetin inhibits the interacting reactions between the substrate and the catalytic residues of the enzyme.

Therefore, the docking of naturally obtained flavonoid hesperetin was successfully carried out with thrombin-like serine protease isolated from the sharp-nosed pit viper (D. acutus) venom. The obtained in silico data suggest that the naturally obtained flavonoid hesperetin has proved to be quite beneficial in inhibiting the deleterious effects of thrombin-like serine protease of the snake venom. The overall effort

Table 1: Docking energies and bond information of hesperetin in thrombin-like snake venom protease of sharp-nosed pit viper (top five results)

\begin{tabular}{lll}
\hline Ligand pose & Energy & H-Bond \\
\hline ligand pose 1 & -5.450000 & 5 \\
ligand pose 2 & -5.200000 & 3 \\
ligand pose 3 & -5.190000 & 3 \\
ligand pose 4 & -5.010000 & 2 \\
ligand pose 5 & -4.960000 & 2 \\
\hline
\end{tabular}




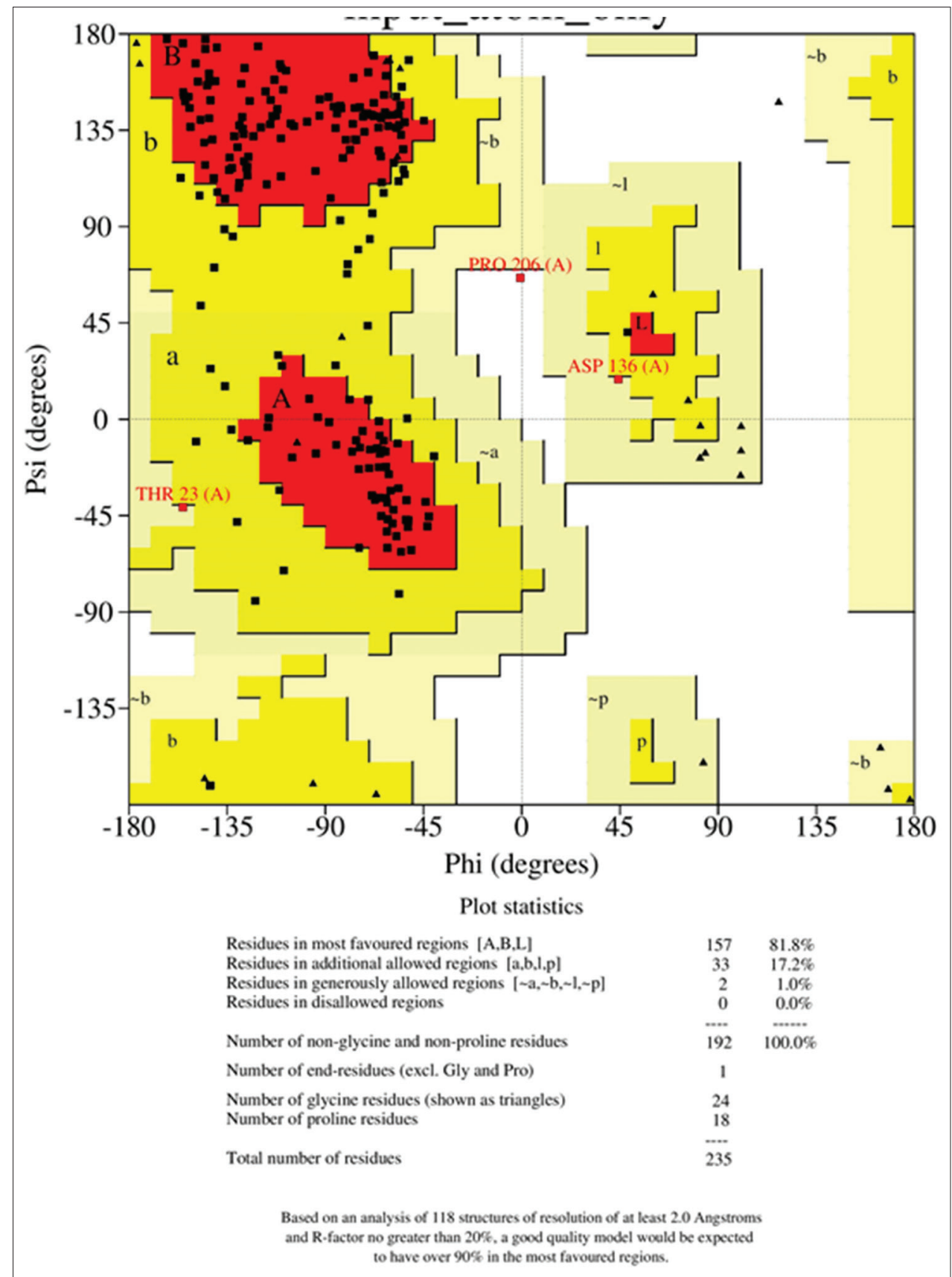

Fig. 1: Ramachandran plot (PROCHECK analysis) of a molecular model of thrombin-like snake venom protease of a sharp-nosed pit viper

of the molecular model and another post-model analysis data revealed the insight of this naturally occurring novel compound to become a futuristic lead molecule, which could be further developed by structural modification for exhibiting more potent activity against snake venom serine proteases.

\section{CONCLUSION}

Recent advancements in the treatment of snake bite envenomation have revealed the crucial role of immunotherapy as life savior agents. However, various adverse effects related to their application cannot be overlooked. Hence, strong interests have been arisen among the researchers and venom toxinologists working in this area to search for other substitute treatment modes to resolve the issues related to snake venom poisoning. Treatment with the indigenous medicinal plants has been proved to be very potential and attractive strategy for the therapy against snake envenomation. Recently, researchers are focusing much on the implementation of natural medicinal plants for treating snakebite poisoning due to their safe, nontoxic, cost-effective, and ubiquitous nature. In our research work, we have implemented the molecular docking of natural compound hesperetin with the thrombin-like serine proteases obtained from the sharp-nosed pit viper (D. acutus) venom. We have implemented the state-of-the-art docking program for validating the hypothesis of the promising inhibitory features of hesperetin on snake venom serine proteases. Hence, the generated in silico results suggest that the novel structure - flavonone might act as a potent inhibitor of thrombin-like snake venom proteases, and unlocks the possibilities for designing drugs of the inhibitors of snake venom serine proteases. Our future efforts would be to further develop this novel natural compound by modification of its functional groups to yield more potent analog for inhibiting thrombin-like serine proteases isolated from snake venom. 


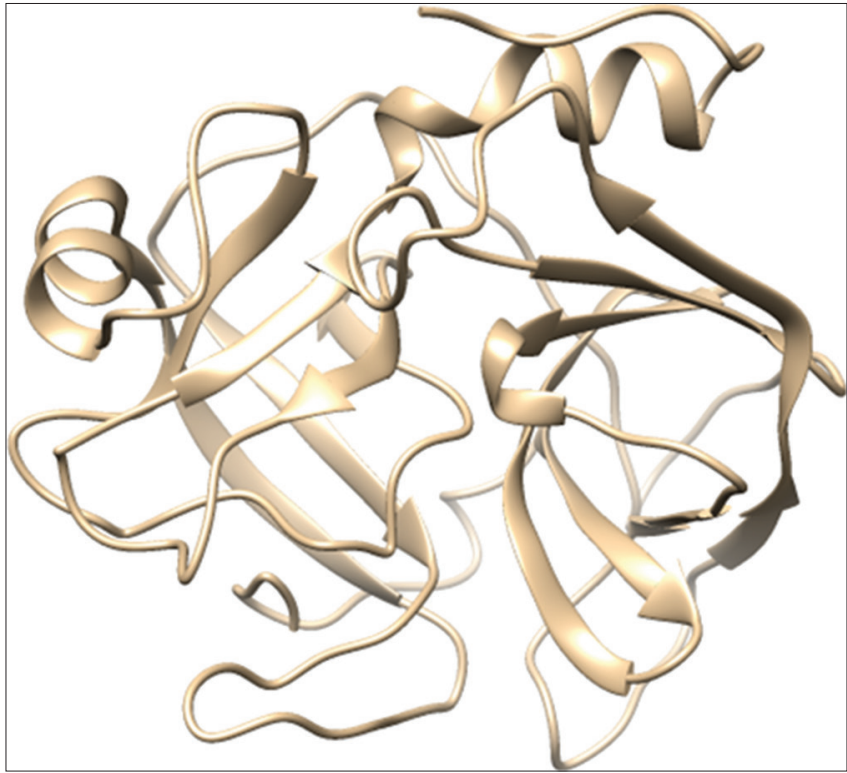

Fig. 2: Three-dimensional modeled structure of thrombin-like snake venom protease of sharp-nosed pit viper

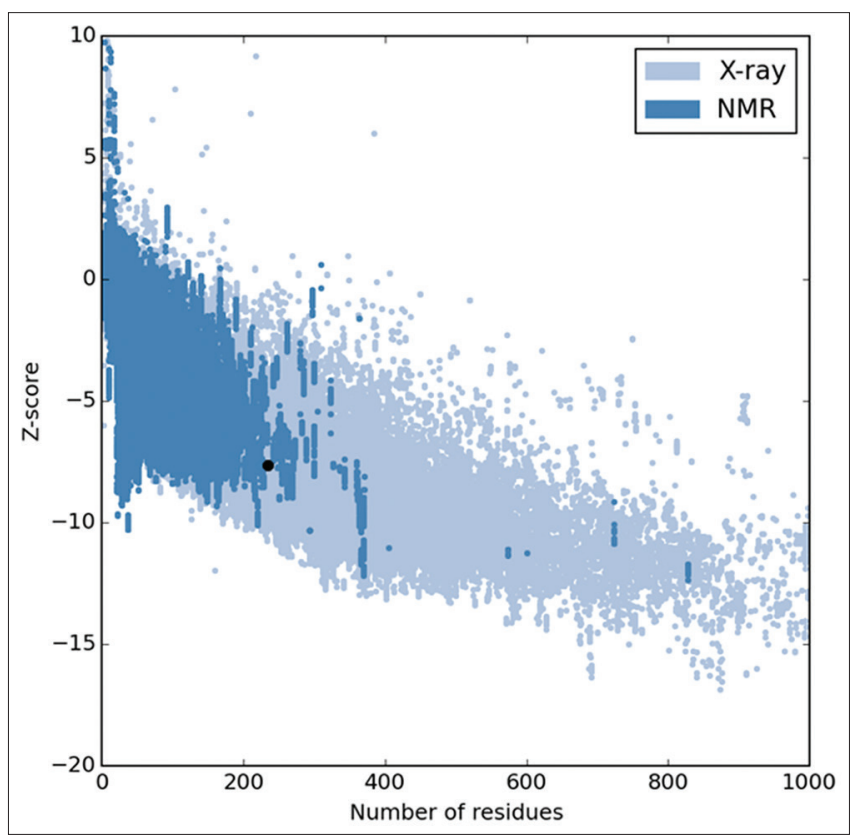

Fig. 3: ProSA-web analysis (stereo-chemical validation) of modeled protein structure of thrombin-like snake venom protease of a sharp-nosed pit viper

\section{ACKNOWLEDGMENT}

We are very thankful to Mrs. Susmita Chakraborty, Chairman, Trinity Trust, Asansol, West Bengal, and Prof. Kalyan Kumar Sen, Principal, Gupta College of Technological Sciences, Asansol, West Bengal, for providing infrastructure facilities for carrying out the research work. Authors are greatly indebted to National Institute of Health and National Center for Biotechnology Information for providing database support.

\section{AUTHOR'S CONTRIBUTION}

Subhamay Panda: Planned, design, carried out the research work and writing of the manuscript. Iman Ehsan: Design of the research and writing of the manuscript.

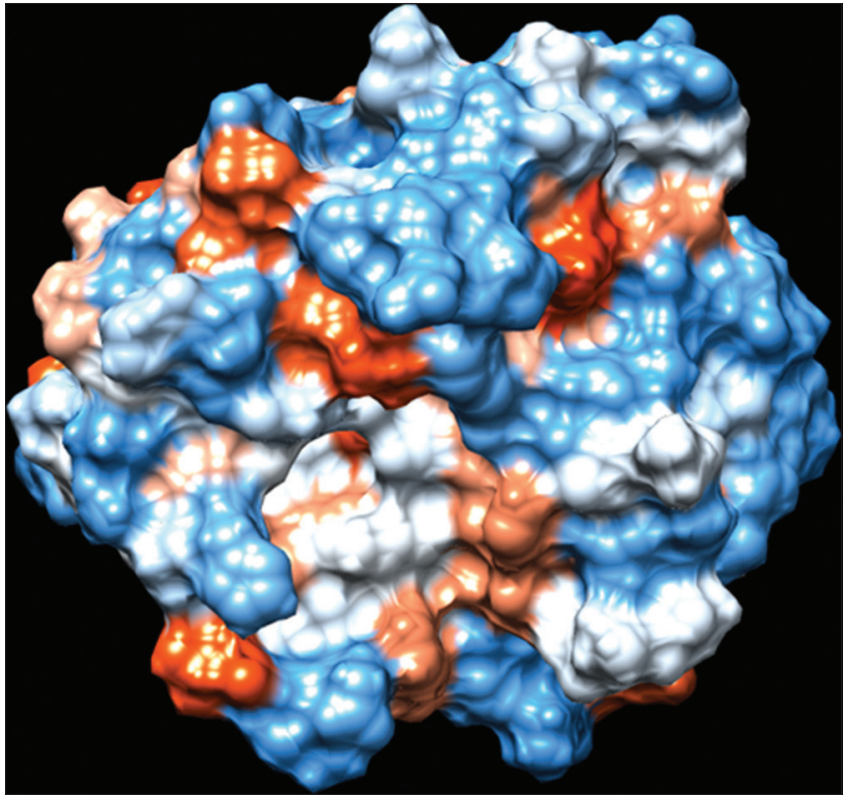

Fig. 4: Hydrophobicity surface maps of thrombin-like snake venom protease of sharp-nosed pit viper (dodger blue for the most hydrophilic, to white, to orange-red for the most hydrophobic)

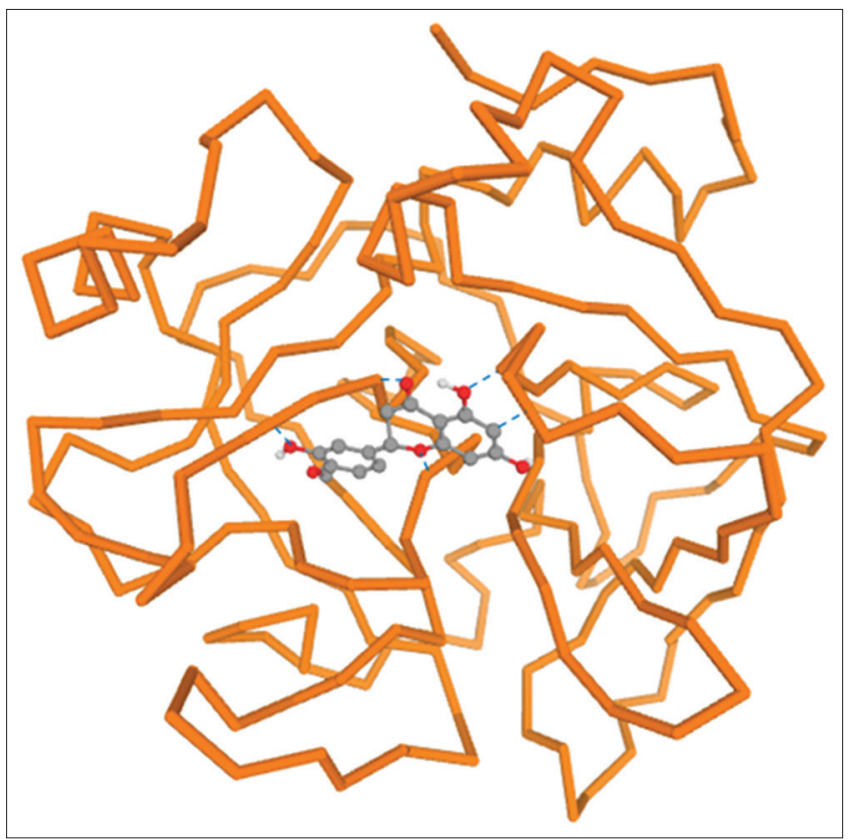

Fig. 5: Molecular docking of hesperetin in thrombin-like snake venom protease of a sharp-nosed pit viper

\section{CONFLICTS OF INTEREST}

The authors do not have any conflicts of interest.

\section{REFERENCES}

1. Lu Q, Clemetson JM, Clemetson KJ. Snake venoms and hemostasis. J Thromb Haemost 2005;3:1791-9.

2. Sanchez EF, Flores-Ortiz RJ, Alvarenga VG, Eble JA. Direct fibrinolytic snake venom metalloproteinases affecting hemostasis: Structural, biochemical features and therapeutic potential. Toxins (Basel) 2017;9.

3. Serrano SM. The long road of research on snake venom serine proteinases. Toxicon 2013;62:19-26.

4. Sousa LF, Portes-Junior JA, Nicolau CA, Bernardoni JL, Nishiyama Jr 
MY., Amazonas DR, et al. Functional proteomic analyses of Bothrops atrox venom reveals phenotypes associated with habitat variation in the Amazon. J Proteomics 2017;159:32-46.

5. Matsui T, Fujimura Y, Titani K. Snake venom proteases affecting hemostasis and thrombosis. Biochim Biophys Acta 2000;1477:146-56.

6. Santiago PB, de Araújo CN, Charneau S, Bastos IMD, Assumpção TCF, Queiroz RML, et al. Exploring the molecular complexity of Triatoma dimidiata sialome. J Proteomics 2018;174:47-60.

7. Tu AT. Overview of snake venom chemistry. Adv Exp Med Biol 1996;391:37-62

8. Inderjit, Dakshini KM. Hesperetin 7-rutinoside (hesperidin) and taxifolin 3-arabinoside as germination and growth inhibitors in soils associated with the weed, Pluchea lanceolata (DC) C.B. Clarke (Asteraceae). J Chem Ecol 1991;17:1585-91.

9. Hussain H, Al-Harrasi A, Abbas G, Rehman NU, Mabood F, Ahmed I, et al. The genus Pluchea: Phytochemistry, traditional uses, and biological activities. Chem Biodivers 2013;10:1944-71.

10. Panda S, Kumari L. Discovery of an unexplored protein structural scaffold of serine protease from big blue octopus (Octopus cyanea): A New prospective lead molecule. Curr Drug Discov Technol 2017;14:135-40.

11. Panda S, Chandra G. Molecular modeling and structural analysis of some snake venom specific toxin proteins and cognate non-toxin proteins of other chordates. Curr Drug Discov Technol 2017;14:59-69.

12. Pantaleão SQ, Philot EA, de Resende-Lara PT, Lima AN, Perahia D, Miteva MA, et al. Structural dynamics of DPP-4 and its influence on the projection of bioactive ligands. Molecules 2018;23.

13. Panda S, Hazra S. Sequence analysis of serum paraoxonase 1 of bathyergidae family specific rats. Int J Pharma Biosci 2016;7:B702-B6.

14. Panda S, Poddar S. A comparative study of Re1-silencing transcription factor (REST) of teleost fishes. Asian J Pharm Clin Res 2017;10:368-71.

15. Panda S, Chandra G. Physicochemical characterization and functional analysis of some snake venom toxin proteins and related non-toxin proteins of other chordates. Bioinformation 2012;8:891-6.

16. Panda S, Chandra G. Sequence analysis and phylogenetic study of some toxin proteins of snakes and related non-toxin proteins of chordates. Bioinformation 2013;9:259-66.

17. Panda S, Kumari L, Panda S. Structural analysis of CD59 of chinese tree shrew: A New reference molecule for human immune system specific CD59 drug discovery. Curr Drug Discov Technol 2017.

18. Vander Dos Santos R, Villalta-Romero F, Stanisic D, Borro L, Neshich G, Tasic L, et al. Citrus bioflavonoid, hesperetin, as inhibitor of two thrombin-like snake venom serine proteases isolated from Crotalus simus. Toxicon 2018;143:36-43.

19. Coordinators NR. Database resources of the National Center for
Biotechnology Information. Nucleic Acids Res 2015;43:D6-17.

20. Petersen TN, Brunak S, von Heijne G, Nielsen H. SignalP 4.0: Discriminating signal peptides from transmembrane regions. Nat Methods 2011;8:785-6.

21. Zhang Y. I-TASSER server for protein 3D structure prediction. BMC Bioinformatics 2008;9:40

22. Guex N, Peitsch MC. SWISS-MODEL and the swiss-pdbViewer: An environment for comparative protein modeling. Electrophoresis 1997:18:2714-23.

23. Laskowski RA, MacArthur MW, Moss DS, Thornton JM. PROCHECK: A program to check the stereochemical quality of protein structures. J Appl Crystallogr 1993;26:283-91.

24. Sippl MJ. Recognition of errors in three-dimensional structures of proteins. Proteins 1993; 17:355-62

25. Pettersen EF, Goddard TD, Huang CC, Couch GS, Greenblatt DM, Meng EC, et al. UCSF chimera-A visualization system for exploratory research and analysis. J Comput Chem 2004;25:1605-12.

26. Morris GM, Huey R, Lindstrom W, Sanner MF, Belew RK, Goodsell DS, et al. AutoDock4 and autoDockTools4: Automated docking with selective receptor flexibility. J Comput Chem 2009;30:2785-91.

27. Sanner MF. Python: A programming language for software integration and development. J Mol Graph Model 1999;17:57-61.

28. Sanner MF, Olson AJ, Spehner JC. Reduced surface: An efficient way to compute molecular surfaces. Biopolymers 1996;38:305-20.

29. Negi SS, Schein CH, Oezguen N, Power TD, Braun W. InterProSurf: A web server for predicting interacting sites on protein surfaces. Bioinformatics 2007;23:3397-9.

30. Labbé CM, Rey J, Lagorce D, Vavruša M, Becot J, Sperandio O, et al. MTiOpenScreen: A web server for structure-based virtual screening. Nucleic Acids Res 2015;43:W448-54.

31. Panfoli I, Calzia D, Ravera S, Morelli A. Inhibition of hemorragic snake venom components: Old and new approaches. Toxins (Basel) 2010;2:417-27.

32. Martz W. Plants with a reputation against snakebite. Toxicon 1992;30:1131-42.

33. Mors WB, Nascimento MC, Pereira BM, Pereira NA. Plant natural products active against snake bite-the molecular approach. Phytochemistry 2000;55:627-42.

34. Alagesaboopathi C. Antimicrobial potential and phytochemical screening of Andrographis affinis Nees an endemic medicinal plant from India. Int J Pharm Pharm Sci 2011;3:157-9.

35. Janardhan B, Shrikanth VM, Dhananjaya BL, More SS. Antisnake venom properties of medicinal plants. Int J Pharm Pharm Sci 2014;7:21-6. 